\title{
Internal Hernia through a Defect in the Broad Ligament of Uterus: Laparoscopic Management Using a Self-Anchoring Barbed Suture
}

\author{
Je Hyung Park, M.D., Soo-Hong Kim, M.D., Yong-Hoon Cho, M.D. \\ Department of Surgery, Pusan National University Yangsan Hospital, Yangsan, Korea
}

\begin{abstract}
The occurrence of internal hernia through a defect in the broad ligament is a very rare condition, which may cause small bowel obstruction. This is a case of a 50-year-old woman who developed intestinal obstruction induced by internal hernia and who had undergone laparoscopic myomectomy 7 years prior to visiting our emergency room. Abdominopelvic computed tomography showed luminal narrowing of the ileum and dilatation of the pelvic loop of the small bowel at the left side of the uterus. We detected internal hernia through the defect in the broad ligament and managed it successfully by performing a laparoscopic procedure using a barbed suture, V-Loc (Covidien, Mansfield, MA).
\end{abstract}

Keywords: Internal hernia, Broad ligament, Small bowel obstruction, Laparoscopy, Barbed suture

\author{
Received January 17, 2018 \\ Accepted February 7, 2018 \\ Corresponding author \\ Soo-Hong Kim \\ Department of Surgery, Pusan \\ National University Yangsan \\ Hospital, 20 Geumo-ro, Mulgeum- \\ eup, Yangsan 50612, Korea \\ Tel: $+82-55-360-2124$ \\ Fax: +82-55-360-2154 \\ E-mail: soohongnara@hanmail.net \\ ORCID: \\ http://orcid.org/0000-0001-7085-5969
}

Copyright @ 2018 The Journal of Minimally Invasive Surgery. All rights reserved.
This is an Open Access article distributed under the terms of the Creative Commons Attribution Non-Commercial License (http:// creativecommons.org/licenses/by-nc/4.0/) which permits unrestricted non-commercial use, distribution, and reproduction in any medium, provided the original work is properly cited.

\section{INTRODUCTION}

Abdominal hernias consist of external and internal hernias. Most abdominal hernias are external hernia, which is related to the absence or weakness of the abdominal wall. Internal hernia is defined as a visceral protrusion through the defect of the peritoneum or mesentery, ${ }^{1}$ accounting for $1 \%$ of reported causes of intestinal obstruction. Paraduodenal hernia (50\%) is the most common type of internal hernia, followed by pericecal hernia (13\%) and hernias through the foramen of Winslow $(8 \%)^{2}$

Hernia through a defect in the broad ligament is a very rare type of internal hernia, accounting for only $4 \%$ of all internal hernias, and is first reported by Quain during autopsy. ${ }^{3}$ Preoperative diagnosis is difficult due to lack of typical signs or symptoms. ${ }^{4}$ Hence, most patients had been diagnosed during surgical exploration. ${ }^{3}$

We report a case of hernia through a defect in the broad ligament, which was preoperatively diagnosed with computed tomography (CT) and successfully managed by laparoscopic procedure using a barbed suture.

\section{CASE REPORT}

A 50-year-old woman visited our emergency room because of lower abdominal pain and vomiting for 3 days. She underwent laparoscopic uterine myomectomy at another hospital 7 years prior to this visit. The patients' vital signs were within normal limits. Abdominal physical examination revealed localized tenderness with tympanitic sound at the lower abdo- 
men. White blood cell and neutrophil counts were increased at $20,690 / \mathrm{ml}$ and $86.6 \%$, respectively, but other laboratory findings were within normal range. On plain abdominal Xray, multiple air-fluid levels were found, and CT scan showed luminal narrowing of the ileum and dilatation of the pelvic loop of the small bowel in the left side of the uterus (Fig. 1). We diagnosed the patient as having hernia through a defect in the broad ligament.

Laparoscopic exploration was immediately performed. With the patient in supine position, three trocars were inserted at the umbilicus $(11 \mathrm{~mm})$, right lower quadrant $(5 \mathrm{~mm})$, and left lower quadrant $(5 \mathrm{~mm})$. The incarcerated distal ileum was found at a 3-cm-sized defect in the left broad ligament (Fig. 2A, B). The incarcerated small bowel revealed mild ischemic change, but because vascularity and motility were recovered after reduction, we did not perform resection. The defect in the broad ligament was closed with a continuous suture using barbed suture, V-Loc 3-0 (Covidien, New Haven, US) (Fig. 2C).

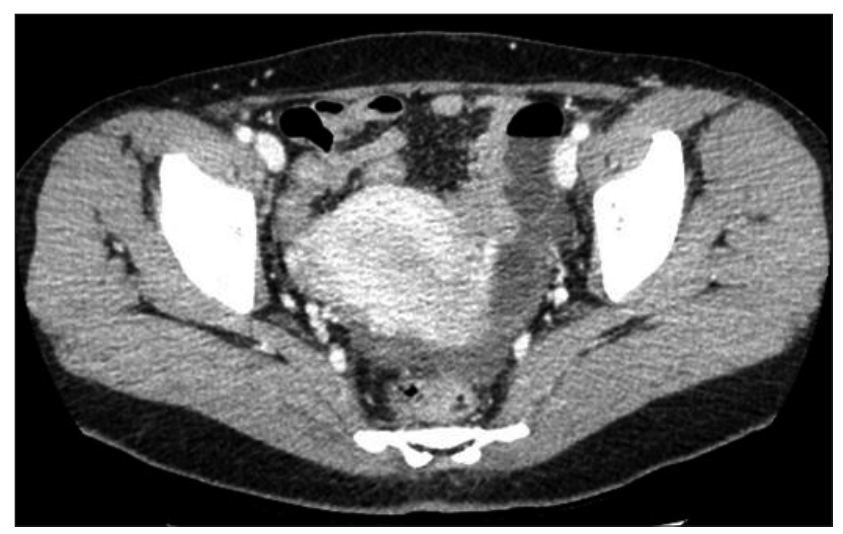

Fig. 1. Abdominopelvic computed tomography shows luminal narrowing of the ileum and pelvic loop dilatation of the small bowel in the left side of the uterus.
The postoperative course was uneventful. At postoperative day (POD) 2, flatus was passed, and at POD 3, diet was started. She was discharged at POD 6. The patient had no complaints after 6 months of follow-up.

\section{DISCUSSION}

The etiology of the defect in the broad ligament is still unknown, but several factors had been suggested. ${ }^{1}$ They were usually considered as two different causes: congenital and acquired. Congenital factors include spontaneous rupture of cystic structures that are remnants of the mesonephric Müllerian ducts and developmental peritoneal defect around the uterus. Acquired factors include operative trauma, pregnancy and birth trauma, and perforations following vaginal manipulation, prior pelvic inflammatory disease, or endometriosis. ${ }^{5,6}$ A typical case of internal hernia through a defect in the broad ligament is reported in middle-aged multi-parous woman without a history of abdominal surgery. ${ }^{7}$ However, the operative trauma, which seemed to occur during laparoscopic uterine myomectomy 7 years prior, could be considered as a possible cause in this case report. To avoid a similar clinical situation, a defect in the broad ligament should be closed when encountered during surgical procedures.

Because this type of internal hernia shows non-specific symptoms and signs, preoperative diagnosis is difficult; hence, some problems may occur before appropriate management. ${ }^{4}$ Making an early diagnosis to prevent complications, such as ischemia, strangulation, and perforation of incarcerated bowels, is important. CT has been known as a useful tool to reveal herniated bowel loops into ectopic locations, and it was also helpful in this case. ${ }^{5,8}$ The herniated bowel loop would be shown as a closed-loop obstruction with C-shaped, U-shaped, or "coffee-bean" configuration, and it ends in close proximity to the uterus. Double-transition zone located in the pelvis, dilated small bowel loops that herniated lateral to the uterus
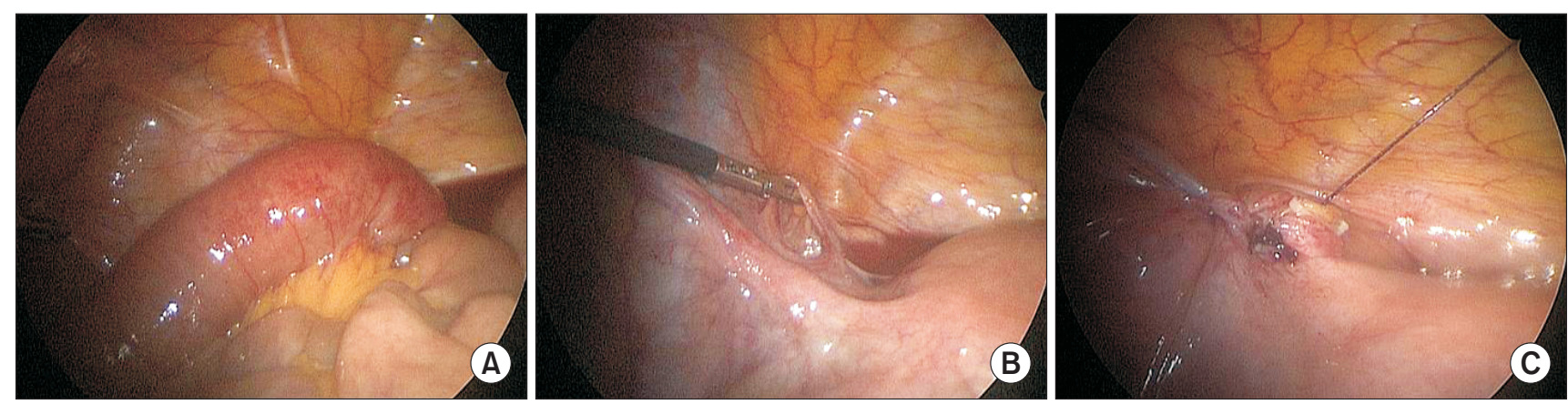

Fig. 2. (A) Intraoperative findings reveal ileal loop herniation through a defect in the left broad ligament. The small bowel has mild ischemic change. (B) After reduction by laparoscopic procedure, the left broad ligament defect was found between the left round ligament and fallopian tube. (C) After closing the defect with V-Loc 3-0. 
in the pelvic cavity, and increased distance between the uterus and ovaries, which are deviated to opposite directions, are also reported in CT findings. ${ }^{8}$ When encountering the small bowel obstruction by unknown origin in female patients, early CT scan would be helpful while considering that internal hernia through a defect in the broad ligament could be one of the possible causes. In our case, CT revealed luminal narrowing of the ileum and pelvic loop dilatation of the small bowel in the left side of the uterus with an anteriorly displaced fallopian tube, and these findings led to early diagnosis.

The treatment for internal hernia through a defect in the broad ligament is surgery, including incarcerated bowel reduction and defect closure. For uncomplicated cases, laparoscopic approach could be thought as a first choice because the reduction and closure are easy even if minimal invasive surgery is tried, and several successful cases have been reported. $2,3,69$ For small bowel obstruction with other internal hernias, laparoscopic approach is accepted as a very technique. ${ }^{3,4}$ Lapa- $^{-}$ roscopic surgery is known to have many advantages, such as decreased infection rate, cosmetic superiority, less pain, and shorter recovery period compared with open surgery. ${ }^{9}$ Closing the defect in the broad ligament sometimes could be problematic without laparoscopic suture and tie skill. However, these problems could be overcome by using a barbed suture material, such as V-Loc. Continuous suture using barbed suture makes a solid suture line easily, without tie. ${ }^{10}$ When internal hernia through a defect in the broad ligament is suspected, prompt laparoscopic approach should be considered to avoid complications and would be helpful to be performed by using a continuous suture with barbed suture material to avoid complications.

When encountering the small bowel obstruction by unknown origin in female patients, early CT scan would be helpful in diagnosing internal hernia through a defect in the broad ligament. In addition, prompt laparoscopic approach using a barbed suture could be helpful in resolving this issue.

\section{ACKNOWLEDGMENTS}

This work was supported by clinical research grant in 2017 from Pusan National University Yangsan Hospital.

\section{REFERENCES}

1) Otani-Takei N, Akimoto T, Sadatomo A, et al. Bowel Obstruction and Peritoneal Dialysis: A Case Report of a Patient with Complications from a Broad Ligament Hernia. Clin Med Insights Case Rep 2016;9:57-60.

2) Matsunami M, Kusanagi H, Hayashi K, Yamada S, Kano N. Broad ligament hernia successfully treated by laparoscopy: Case report and review of literature. Asian J Endosc Surg 2014;7:327-329.

3) Varela GG, Lopez-Loredo A, Garcia Leon JF. Broad ligament hernia-associated bowel obstruction. JSLS 2007;11:127-130.

4) Vyrdal CD, El-Hussuna A. Laparoscopically treated small bowel obstruction due to internal herniation through the broad ligament. Pol Przegl Chir 2014;86:377-379.

5) Quiroga S, Sarrias M, Sanchez JL, Rivero J. Small bowel obstruction secondary to internal hernia through a defect of the broad ligament: preoperative multi-detector CT diagnosis. Abdom Imaging 2012;37:1089-1091.

6) Bangari R, Uchil D. Laparoscopic management of internal hernia of small intestine through a broad ligament defect. J Minim Invasive Gynecol 2012;19:122-124.

7) Barbier Brion B, Daragon C, Idelcadi O, Mantion G, Kastler B, Delabrousse E. Small bowel obstruction due to broad ligament hernia: computed tomography findings. Hernia 2011;15:353-355.

8) Haku T, Daidouji K, Kawamura H, Matsuzaki M. Internal herniation through a defect of the broad ligament of the uterus. Abdom Imaging 2004;29:161-163.

9) Cho S, Yun S. The Usefulness of Laparoscopy in the Treatment of Incarcerated Internal Hernia through a Defect in the Broad Ligament. J Minim Invasive Surg 2015;18:133-136.

10) Patri P, Beran C, Stjepanovic J, Sandberg S, Tuchmann A, Christian H. V-Loc, a new wound closure device for peritoneal closure--is it safe? A comparative study of different peritoneal closure systems. Surg Innov 2011;18:145-149. 